\title{
Study on the Distribution and Source of Multidrug-resistant Organisms Infection in Non-ICU Departments
}

Zhanjie Li ( 18052106999@163.com)

Jiangsu Province Hospital and Nanjing Medical University First Affiliated Hospital https://orcid.org/0000-0002-0703-3230

Xing Wu

affiliated hospital of jiangnan university

Bo Liu

first affiliated hospital of nanjing medical university

Wei-hong Zhang

jiangsu shengze hospital affiliated to nanjing medical university

Su-ming Zhou

first affiliated hospital of nanjing medical university

Yong-xiang Zhang

first affiliated hospital of nanjing medical university

\section{Research}

Keywords: multidrug-resistant organisms, non-ICU, distribution, source

Posted Date: February 22nd, 2021

DOI: https://doi.org/10.21203/rs.3.rs-215930/v1

License: (c) (1) This work is licensed under a Creative Commons Attribution 4.0 International License. Read Full License 


\section{Abstract}

Objective: To analyze the distribution and source of multidrug-resistant organisms (MDRO) infection in non-ICU departments, and provide basis for accurate prevention and control measures of MDRO.

Methods: A total of 1116 MDRO infection strains isolated from 802 patients with MDRO infection in the non-ICU departments of The First Affiliated Hospital with Nanjing Medical Universityl from October 2017 to September 2019 were selected as subjects. According to the source, MDRO was divided into two types and four groups: out-of-hospital infection (transferred from outside hospital and community-acquired) and nosocomial infection (transferred from our hospital and department-acquired).

Results: The major MDRO infections in non-ICU departments were CRE (34.41\%) and MRSA (29.66\%). There were significant differences in the composition of MDRO infection among different departments $(X 2=185.687, P<0.001)$. The departments with the most MDRO infection were geriatric medicine (13.17\%), neurosurgery (10.04\%), and rehabilitation medicine (8.51\%). The detection rates of CRE, MRSA, CRAB, and CRPA were $10.69 \%, 43.83 \%, 33.72 \%$, and $27.11 \%$, respectively, which were significantly lower than those of CRE, MRSA, CRAB, and CRPA in the departments of the whole hospital (25.35\%, $51.48 \%, 79.15 \%$, $46.99 \%)$, and the differences were statistically significant $(\chi 2=584.309,15.583,960.632,203.726$; all $P<0.001)$. There were significant differences in the detection rates of four kinds of MDRO among different non-ICU departments $(\chi 2=190.766,97.642,75.078,69.515$; all $P<0.001)$. The most common sites of MDRO infection were lower respiratory tract (48.39\%), surgical site (11.83\%), and urinary tract (11.02\%). There were 641 cases of out-of-hospital infection (57.44\%), including out-of-hospital transfer (33.42\%) and community-acquire (24.01\%), and 475 cases of nosocomial infection (42.56\%), including our hospital transfer (4.66\%) and department-acquire (37.90\%). The composition of MDRO source was different among non-ICU departments.

Conclusion: Nearly 2/3 of MDRO infection in non-ICU departments came from out-of-hospital input and in-hospital transfer. The implementation of basic infection control measures and early identification through information system need be noticed. The distribution and source of MDRO infection varied in different departments, and targeted prevention and control measures should be made according to its characteristics to achieve accurate prevention and control.

\section{Introduction}

The spread of multidrug-resistant organisms (MDRO) has become a prominent public health issue worldwide. Effective treatment for MDRO infection is often limited, which leads to additional morbidity and mortality and increased medical expenses, posing enormous burden to patients and medical systems. Therefore, controlling the spread of MDRO is concerned with great public interest ${ }^{[1-4]}$. ICU patients become the high-risk group of MDRO infection due to the severe condition, invasive operations, low immunity, and antimicrobial exposure. Therefore, the researches on MDRO at home and abroad mainly focus on ICU

${ }^{[6-8]}$. In recent years, with the increasing mobility and crossover of patients among medical institutions and departments in the hospital, as well as the more and more extensive use of antibiotics, MDRO infection in non-ICU departments has also increased significantly. However, the current domestic research on MDRO infection in non-ICU departments is extremely rare. In which non-ICU departments does MDRO infection mainly occur? What are the sources of these MDRO? Whether there exist differences in the distribution and source of different MDRO infection in non-ICU departments? We need to think about and explore these issues. Only in this way can we have targeted and precise prevention and control of MDRO infection. This study retrospectively analyzed the distribution and source of MDRO infection in non-ICU departments, with the aim to provide reference for other medical institutions.

\section{Materials And Methods}

\section{Clinical data}

A total of 802 patients with MDRO infection in all non-ICU departments in The First Affiliated Hospital with Nanjing Medical University from October 2017 to September 2019 were selected, including 529 males (65.96\%) and 273 females (34.04\%), with an average age of $58 \pm 21$ years from 1 day to 100 years. Inclusion: all patients in non-ICU departments with MDRO infection. Exclusion: ICU patients, patients without MDRO detection, or patients with MDRO detection but colonization or contamination. The repetitive strains at the same site of the same patient were excluded. This study was approved by the Ethics Committee of The First Affiliated Hospital with Nanjing Medical University (2019-SR-075).

\section{Research method}

Through the Nosocomial Infections Surveillance System (Xinglin-tech, Hangzhou, Zhejiang, China), we recruited 802 MDRO infected inpatients from non-ICU departments of our hospital from October 2017 to September 2019, and isolated 1116 MDRO infected strains, including: methicillin-resistant staphylococcus aureus (MRSA), carbapenem resistant enterobacteriaceae (CRE), carbapenem resistant acinetobacter baumannii (CRAB), carbapenem resistant pseudomonas aeruginosa (CRPA). The professional staff of nosocomial infection consulted each infection case record item by item, and divided the sources into two types and four groups: out-of-hospital infection (transferred from outside hospital and community-acquired) and nosocomial infection (transferred from our hospital and department-acquired), thereby exploring the distribution and source of MDRO infection.

\section{Diagnostic criteria for infection}

The diagnostic criteria for infection adopted the Diagnostic Criteria for Nosocomial Infection (Trial) issued by Ministry of Health in $2001{ }^{[9]}$. The criteria for MDRO was based on the consensus of international experts ${ }^{[10]}$ 


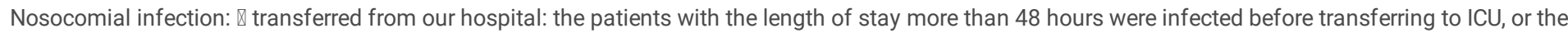
infection occurred within 48 hours after transferring to ICU in other departments of our hospital; $\mathbb{\nabla}$ department-acquired: infection occurred during hospitalization in ICU 48 hours after transfer to ICU, or infection within 48 hours after transfer from ICU; out-of-hospital infection: 囚: transferred from outside hospital: infection occurred more than 48 hours after hospitalization in other hospitals; $₫$ community-acquired: no hospitalization in other hospitals, or the infection occurred within less than 48 hours of hospitalization in other hospitals. MDRO detection rate $=$ the number of MDRO strains detected (including infection and colonization)/total number of bacteria detected in the same period $\times 100 \%$.

\section{Bacterial identification and drug sensitivity test}

VITEK-2Compact automatic bacteria identification instrument (bioMérieux S.A., France) or API identification system (bioMérieux S.A., France) were used for bacteria identification. VITEK-2Compact automatic bacterial identification instrument or Kirby-Bauer test (Oxide, Japan) was used for bacterial drug sensitivity test. The results of drug sensitivity were interpreted according to the Clinical and Laboratory Standards Institute (2019) ${ }^{[11]}$. The quality-control strains (National Center for Clinical Laboratories) included escherichia coli (25922; American Type Culture Collection, ATCC, USA), klebsiella pneumoniae (700603; ATCC), staphylococcus aureus (25923; ATCC), acinetobacter baumannii (19606; ATCC), pseudomonas aeruginosa (278553; ATCC) and enterobacter cloacae (700323; ATCC).

\section{Statistical analysis}

SPSS 20.0 (IBM Corp., USA) and GraphPad Prism 7.0 (GraphPad Software, USA) were used for statistical analysis. MDRO infection site, distribution, and detection rate in each department were enumeration data, which were described as constituent ratio and rate. The comparison among multiple groups was performed using $\chi 2$ test. The stacked plot was drawn according to the MDRO infection source. $P \llbracket 0.050$ meant statistical significance.

\section{Results}

\section{MDRO distribution}

A total of 1116 MDRO strains were detected in non-ICU departments, mainly CRE (34.41\%) and MRSA (29.66\%). There were significant differences in MDRO infection among different departments $(P<0.001)$. The departments with the most MDRO infections were geriatric medicine $(13.17 \%)$, neurosurgery $(10.04 \%)$, and rehabilitation medicine (8.51\%). As for the proportion of different MDRO infections in departments, the proportion of CRE infection was high in hepatobiliary center, urinary surgery, and pancreaticobiliary center ( $\geq 50 \%$ ); the proportion of MRSA infection was high in plastic and burn surgery (49.09\%) and orthopedics (39.22\%); the proportion of CRAB infection was high in orthopedics (43.14\%); the proportion of CRPA infection was high in respiratory department (38.00\%). The details were shown in Table 1 and Figure 1.

\section{MDRO detection rate}

The detection rates of CRE, MRSA, CRAB and CRPA in non-ICU departments were $10.69 \%, 43.83 \%, 33.72 \%$ and $27.11 \%$ respectively, which were significantly lower than those in the whole hospital $(25.35 \%, 51.48 \%, 79.15 \%$ and $46.99 \%)$ (all $P<0.001)$. Among them, the neurosurgery $(26.24 \%)$ and rehabilitation medicine (25.50\%) had higher detection rate of CRE; pancreaticobiliary center (86.11\%), neurosurgery $(70.45 \%)$, rehabilitation medicine $(60.71 \%)$, and geriatric medicine (59.22\%) had higher detection rate of MRSA; hepatobiliary center (65.79\%), rehabilitation medicine (58.93\%) and pancreaticobiliary center (53.85) had higher detection rate of CRAB; hepatobiliary center (42.42\%) had higher detection rate of CRPA. There were significant differences in the detection rates of 4 kinds of MDRO infections among different non-ICU departments (all $P<0.001$ ). The details were shown in Table 2.

\section{MDRO infection sites}

The most infected sites of MDRO in non-ICU departments were lower respiratory tract (48.39\%), surgical site (11.83\%), and urinary tract (11.02\%). The details were shown in Table 3.

\section{MDRO source}

Of the 1116 MDRO strains isolated from the patients in non-ICU departments, 641 (57.44\%) were out-of-hospital infection including transferred from outside hospital (33.42\%) and community-acquired (24.01\%), and 475 (42.56\%) were nosocomial infection including transferred from our hospital (4.66\%) and department-acquired (37.90\%). The composition of MDRO source was different among non-ICU departments. The proportions of out-of-hospital infection of CRE (Fig. 2a), MRSA (Fig. 2b), CRAB (Fig. 2c) and CRPA (Fig. 2d) were 52.34\%, 63.75\%, 49.55\% and 65.36\% respectively, among which 34.64\%, 33.53\%, 28.83\% and $36.31 \%$ were transferred from outside hospital, and $8.23 \%, 30.21 \%, 20.72 \%$ and $29.05 \%$ were community-acquired; the proportions of nosocomial infection were $47.66 \%, 36.25 \%, 50.45 \%$ and $34.64 \%$ respectively, among which $6.25 \%, 1.51 \%, 9.01 \%$ and $1.68 \%$ were transferred from our hospital, and $40.89 \%$, $34.74 \%, 41.44 \%$ and $32.96 \%$ were department-acquired. Nearly $80 \%$ of CRE infection in rehabilitation department was transferred from outside hospital, and nearly $70 \%$ of CRE infection in geriatric medicine department was department-acquired. CRE infection in general practice medicine department, nephrology department, urinary surgery department, and neurosurgery department was mostly out-of-hospital infection, while CRE infection in geriatric medicine department, hepatobiliary center, and pancreaticobiliary center was mainly nosocomial infection. Similarly, the sources of MRSA, CRAB, and CRPA varied in different departments. The details were shown in Table 2.

\section{Discussion}


This study showed that CRE (34.41\%) and MRSA (29.66\%) were the main MDRO infections in non-ICU departments, while CRAB (64.80\%) and CRE (20.09\%) were the main MDRO infections in ICU department, indicating that MDRO infection in non-ICU departments had certain particularity, which is similar to the results of Zhang et al. ${ }^{[14]}$. The reason might be that the average length of stay of patients in ICU ward was longer and invasive operation was more, which led to CRAB detection in ICU department significantly higher than that in non-ICU departments. Consistent with the results of Long et al. ${ }^{[15]}$, geriatric medicine, neurosurgery, and rehabilitation medicine were the three departments with the most MDRO infections in non-ICU departments, which were related to the characteristics of patients and diseases in the three departments, and should be paid more attention in the prevention and control of MDRO. In addition, this study found that the proportions of four kinds of MDRO infection varied in different non-ICU departments, and consequently prevention and control measures should be taken according to the "main contradiction" of MDRO infection in each department. In this study, the detection rates of CRE, MRSA, CRAB, and CRPA in non-ICU departments were $10.69 \%, 43.83 \%, 33.72 \%$, and $27.11 \%$ respectively, which were higher than the results of relevant studies ${ }^{[14,16]}$. Meanwhile, compared with the corresponding data of non-ICU departments in the China Antimicrobial Resistance Surveillance System (CARSS) in 2018 (8\%, 28\%, 43\% and $20 \%{ }^{[17]}$, the detection rates of MRSA and CRE in this study were more prominent. The departments with the highest detection rate of MRSA were pancreaticobiliary center (86.11\%), neurosurgery (70.45\%) and rehabilitation medicine $(60.71 \%)$, while the departments with high detection rate of CRE were neurosurgery (26.24\%) and rehabilitation medicine (25.50\%). MRSA infection leads to 20000 deaths of hospitalized patients in the USA every year ${ }^{[18]}$, and CRE causes 20 billion US dollars of medical expenses and 23000 deaths of antibiotic resistant bacteria every year ${ }^{[19]}$, which should be paid special attention. In this study, the top three MDRO infection sites in non-ICU departments were lower respiratory tract (48.39\%), surgical site (11.83\%), and urinary tract (11.02\%), which were significantly different from the data of ICU ward [lower respiratory tract (85.04\%), blood flow (4.69\%) and surgical site (3.79\%)] [12], suggesting that we should focus on the surgical site infection and urinary tract infection caused by MDRO in non-ICU departments.

The out-of-hospital infection of MDRO in non-ICU departments was $57.44 \%$, and the nosocomial infection was $42.56 \%$, which was different from the data in ICU (out-of-hospital infection $50.89 \%$ and nosocomial infection $49.11 \%$ ). The proportion of out-of-hospital infection in non-ICU departments was higher, among which the proportions of transferred from outside hospital, community-acquired, transferred from our hospital and department-acquired were $33.42 \%$, $24.01 \%, 4.66 \%$ and $37.90 \%$, respectively. That is to say, only about $1 / 3$ of MDRO infections were department-acquired, and nearly $2 / 3$ of MDRO infections were imported from other hospital or transferred from other departments in our hospital. At present, the domestic MDRO patients after discharge lack of follow-up, tracking, and reminder mechanism and measures, which may bring about cross infection between medical institutions and communities after the MDRO patients transfer or return to the community. A previous study ${ }^{[20]}$ has shown that the proportion of patients carrying MDRO within one year after discharge from ICU is very high, which highlights the ability of MDRO to persist in susceptible patients and form a communication library when they are readmitted. Moreover, MDRO is found in many community environments ${ }^{[21]}$, which also confirms the spread of MDRO caused by poor management after discharge.

The identification of persistent carriers at readmission has become an integral part of the program aimed at controlling the spread of MDRO in medical facilities ${ }^{[22]}$. Meanwhile, the implementation of handover measures for patients with MDRO is also crucial when they are transferred to other departments. Haverkate et al. ${ }^{[23]}$ support the use of automatic alerts based on hospital information system to prevent the transmission of MDRO between department transfer. Octaria Rany et al. ${ }^{[24]}$ suggest to design and establish a network platform at the whole social level (connecting medical institutions and communities) to achieve effective identification and early prevention and control of MDRO patients.

It has been reported that the pandemic of COVID-19 has made people aware of the importance of basic infection measures, including hand washing, equipment cleaning, and the use of PPE in providing patient care ${ }^{[25]}$, and these measures can also effectively reduce the incidence of MDRO nosocomial infection.

This study demonstrated that nearly $2 / 3$ of MDRO infections in non-ICU departments came from out-of-hospital and in-hospital transfer. Attention should be paid to the implementation of basic measures for infection control and the early identification of infection through information system. Moreover, the distribution and source of MDRO infection varied in different departments, so we should formulate targeted prevention and control measures according to their characteristics to achieve accurate prevention and control.

\section{Declarations}

\section{Funding}

This project was supported by the National Key R \& D Program Fund (2020YFC0848100), the Research Foundation for Infection Prevention and Control of Chinese Geriatrics Association (GRYJ-LRK2018016), and the Jiangsu Provincial Hospital Management Innovation Research Fund (JSYGY-3-2019-484).

\section{Acknowledgments}

We thank Xing Wu and Ge Song for their contributions to data collection in this research.

\section{Conflict of Interest}

The authors have no conflicts of interest to declare.

\section{Ethics Statement}

The authors are accountable for all aspects of the work with regard to ensuring that questions related to the accuracy or integrity of any part of the work are appropriately investigated and resolved. This study was approved by Institutional Review Board (No. 2019-SR-075), and each hospital received permission to participate in this study and sign a cooperation agreement. Written informed consent was obtained from all patients. 


\section{References}

1. Wijnakker R, Lambregts M M C, Rump B et al. Limited multi-drug resistant organism related stigma in carriers exposed to isolation precautions: an exploratory quantitative questionnaire study [J]. J Hosp Infect, 2020, 106: 126-133.

2. Anaya-Baz Blanca, Maldonado Natalia, Palacios-Baena Zaira R et al. Systematic literature review of the burden and outcomes of infections due to multidrug-resistant organisms in Europe: the ABOUT-MDRO project protocol [J]. BMJ Open, 2020, 10: e030608.

3. Huang Y S, Lai L C, Chen Y A, et al. Colonization With Multidrug-Resistant Organisms Among Healthy Adults in the Community Setting: Prevalence, Risk Factors, and Composition of Gut Microbiome [J]. Frontiers in Microbiology, 2020, 11:1402.

4. Huang Jing, Cui Can, Zhou Shuli et al. Impact of multicenter unified enhanced environmental cleaning and disinfection measures on nosocomial infections among patients in intensive care units [J]. J Int Med Res, 2020, 48: 300060520949766.

5. Guzmán-Herrador B, Díaz Molina C, Allam MF, et al. Underlying illness severity and outcome of nosocomial pneumonia: prospective cohort study in intensive care unit [J]. J Hosp Infect, 2014, 86: 53-56区

6. Li Wang, Xiaolong Huang, Jiating Zhou, et al. Predicting the occurrence of multidrug-resistant organism colonization or infection in ICU patients: development and validation of a novel multivariate prediction model [J]. Antimicrob Resist Infect Control. 2020 May 19; 9 (1): 66.

7. Yiang Giou-Teng, Tzeng I-Shiang, Shui Hao-Ai et al. Early Screening of Risk for Multi-Drug Resistant Organisms in the Emergency Department In Patients With Pneumonia And Early Septic Shock: Single-Center, Retrospective Cohort Study [J]. Shock, 2020 Jul 15.

8. Jinru Yang, Dan Liu, Yibin Tan, Likai Lin, Ying Wang. Network path analysis on risk factors for multidrug-resistant organism infection in intensive care unit [J]. Chinese Journal of Infection Control. 2020,19 (02): 148-154.

9. Ministry of Health of the PRC. Diagnostic Criteria for Nosocomial Infection (Trial) [J]. Chinese Medical Journal. 2001,81 (5): $314-320$.

10. Magiorakos AP, Srinivasan A, Carey RB, et al. Multidrug-resistant, extensively druresistant and pandrug-resistant bacteria\an international expert proposal for interim standard definitions for acquired resistance [J]. Clin Microbiol Infect, 2012, 18 (3): 268-281.

11. Clinical and Laboratory Standards Institute (CLSI). Performance standards for antimicrobial susceptibility testing [S]. Twenty seventh informational supplement, 2019.

12. Zhanjie Li, Bo Liu, Huifen Li, et al. Study on distribution and source of multi-drug resistant bacteria infection in ICUs [J]. Chinese Journal of Nosocomiology, 2019, 29 (8): 1166-1171.

13. Feng Zang, Yongxiang Zhang, Bo Liu, et al. Distribution and sources of elderly intensive care unit patients with multidrug-resistant organisms infections [J]. Chinese Journal of Nosocomiology, 2020, 30 (06): 866-871.

14. Yunyan Zhang. Distribution and Drug Resistance of Multidrug Resistant Bacteria in ICU and Non ICU Wards [J]. China Continuing Medical Education, 2019,11 (36): 70-74

15. Shengshuang Long, Xiaoyun Hu, Qipeng Zhang, Guiying Qin, Longmin Qiu. Department distribution of multidrug-resistant organisms and the infection control of some hospital [J]. Chinese Journal of Disinfection, 2016, 33 (10): 986-988.

16. Xuan Zhou, Guiqin Du, Yajun Li, Xiaoxia Chen, Huihong Qu. Difference in detection results and antimicrobial resistance of multidrug-resistant organisms in intensive care unit and non-intensive care unit [J]. Chinese Journal of Infection Control, 2018,17 (03): 219-223 + 229.

17. China Antimicrobial Resistance Surveillance System (CARSS). National bacterial resistance surveillance report in 2018 [OL]. http://www.carss.cn/Report/Details?ald=648.

18. Shariati Aref, Dadashi Masoud, Chegini Zahra et al. The global prevalence of Daptomycin, Tigecycline, Quinupristin/Dalfopristin, and Linezolid-resistant Staphylococcus aureus and coagulase-negative staphylococci strains: a systematic review and meta-analysis [J]. Antimicrob Resist Infect Control, 2020, 9: 56.

19. Predic Marko, Delano John P, Tremblay Elizabeth et al. Evaluation of patient risk factors for infection with carbapenem-resistant Enterobacteriaceae [J]. Am J Infect Control, 2020, 48: 1028-1031.

20. Hope D, Ampaire L, Oyet C. Muwanguzi EAntimicrobial resistance in pathogenic aerobic bacteria causing surgical site infections in Mbarara regional referral hospital, Southwestern Uganda. Sci Rep. 2019 Nov 21; 9 (1): 17299.

21. Jones Makoto, Ying Jian, Huttner Benedikt et al. Relationships between the importation, transmission, and nosocomial infections of methicillin-resistant Staphylococcus aureus: an observational study of 112 Veterans Affairs Medical Centers [J]. Clin Infect Dis, 2014, 58: 32-9.

22. Lucet Jean-Christophe, Koulenti Despoina, Zahar Jean-Ralph, Persistence of colonisation with MDRO following discharge from the ICU [J]. Intensive Care Med, 2014, 40: 603-5.

23. Haverkate Manon R, Derde Lennie P G, Brun-Buisson Christian et al. Duration of colonization with antimicrobial-resistant bacteria after ICU discharge [J]. Intensive Care Med, 2014, 40: 564-71.

24. Octaria Rany, Chan Allison, Wolford Hannah et al. Web-Based Interactive Tool to Identify Facilities at Risk of Receiving Patients with Multidrug-Resistant Organisms [J]. Emerg Infect Dis, 2020, 26: 2046-2053.

25. Cole Jennifer,Barnard Emily, The Impact of the COVID-19 Pandemic on Healthcare Acquired Infections with Multidrug Resistant Organisms [J]. Am J Infect Control, 2020, Available online 2 October.

\section{Tables}


Table 1 Distribution and constituent ratio of MDRO infection in non-ICU departments ( $n \%)$

\begin{tabular}{|c|c|c|c|c|c|c|c|}
\hline Department & CRE & MRSA & CRAB & CRPA & Total & $x^{2}$ & $P$ \\
\hline Geriatric medicine & $42(28.57)$ & $42(28.57)$ & $22(14.97)$ & $41(27.89)$ & $147(13.17)$ & 185.687 & $<0.001$ \\
\hline Neurosurgery & $37(33.04)$ & $23(20.54)$ & $26(23.21)$ & $26(23.21)$ & $112(10.04)$ & & \\
\hline Rehabilitation medicine & $45(47.37)$ & $13(13.68)$ & $17(17.89)$ & $20(21.05)$ & $95(8.51)$ & & \\
\hline Hepatobiliary center & $38(55.07)$ & $2(2.90)$ & $18(26.09)$ & $11(15.94)$ & $69(6.18)$ & & \\
\hline Nephrology & $22(36.07)$ & $18(29.51)$ & $16(26.23)$ & $5(8.20)$ & $61(5.47)$ & & \\
\hline Plastic and burn surgery & $8(14.55)$ & $27(49.09)$ & $13(23.64)$ & $7(12.73)$ & $55(4.93)$ & & \\
\hline Pancreaticobiliary center & $26(50.00)$ & $13(25.00)$ & $10(19.23)$ & $3(5.77)$ & $52(4.66)$ & & \\
\hline Orthopedics & $8(15.69)$ & $20(39.22)$ & $22(43.14)$ & $1(1.96)$ & $51(4.57)$ & & \\
\hline Urinary surgery & $34(66.67)$ & $7(13.73)$ & $5(9.80)$ & $5(9.80)$ & $51(4.57)$ & & \\
\hline Respiratory & $11(22.00)$ & $11(22.00)$ & $9(18.00)$ & $19(38.00)$ & $50(4.48)$ & & \\
\hline Other non-ICU departments & $113(30.29)$ & $155(41.55)$ & $64(17.16)$ & $41(10.99)$ & $373(33.42)$ & & \\
\hline Total & $384(34.41)$ & $331(29.66)$ & $222(19.89)$ & $179(16.04)$ & $1116(100 \%)$ & & \\
\hline
\end{tabular}

Table 2 Detection rate of MDRO in non-ICU departments

\begin{tabular}{|c|c|c|c|c|c|c|c|c|c|c|c|c|}
\hline \multirow[t]{2}{*}{ Department } & \multicolumn{3}{|l|}{ CRE } & \multicolumn{3}{|l|}{ MRSA } & \multicolumn{3}{|l|}{ CRAB } & \multicolumn{3}{|l|}{ CRPA } \\
\hline & Strains & $\begin{array}{l}\text { Detected } \\
\text { strains }\end{array}$ & $\begin{array}{l}\text { Detection } \\
\text { rate }(\%)\end{array}$ & Strains & $\begin{array}{l}\text { Detected } \\
\text { strains }\end{array}$ & $\begin{array}{l}\text { Detection } \\
\text { rate }(\%)\end{array}$ & Strains & $\begin{array}{l}\text { Detected } \\
\text { strains }\end{array}$ & $\begin{array}{l}\text { Detection } \\
\text { rate (\%) }\end{array}$ & Strains & $\begin{array}{l}\text { Detected } \\
\text { strains }\end{array}$ & $\begin{array}{l}\text { Detec } \\
\text { rate ( }\end{array}$ \\
\hline Geriatric medicine & 1350 & 179 & 13.26 & 255 & 151 & 59.22 & 311 & 90 & 28.94 & 669 & 228 & $34.0 \varepsilon$ \\
\hline Neurosurgery & 221 & 58 & 26.24 & 44 & 31 & 70.45 & 85 & 39 & 45.88 & 119 & 45 & 37.82 \\
\hline $\begin{array}{l}\text { Rehabilitation } \\
\text { medicine }\end{array}$ & 298 & 76 & 25.50 & 28 & 17 & 60.71 & 56 & 33 & 58.93 & 110 & 38 & $34.5 ?$ \\
\hline $\begin{array}{l}\text { Hepatobiliary } \\
\text { center }\end{array}$ & 345 & 48 & 13.91 & 14 & 3 & 21.43 & 38 & 25 & 65.79 & 33 & 14 & 42.42 \\
\hline Nephrology & 401 & 26 & 6.48 & 67 & 18 & 26.87 & 53 & 21 & 39.62 & 49 & 6 & 12.24 \\
\hline $\begin{array}{l}\text { Plastic and burn } \\
\text { surgery }\end{array}$ & 81 & 12 & 14.81 & 89 & 29 & 32.58 & 30 & 14 & 46.67 & 45 & 8 & $17.7 \varepsilon$ \\
\hline $\begin{array}{l}\text { Pancreaticobiliary } \\
\text { center }\end{array}$ & 255 & 34 & 13.33 & 36 & 31 & 86.11 & 26 & 14 & 53.85 & 43 & 7 & $16.2 \varepsilon$ \\
\hline Orthopedics & 109 & 10 & 9.17 & 83 & 25 & 30.12 & 53 & 27 & 50.94 & 18 & 1 & 5.56 \\
\hline Urinary surgery & 672 & 40 & 5.95 & 19 & 9 & 47.37 & 27 & 7 & 25.93 & 66 & 6 & 9.09 \\
\hline $\begin{array}{l}\text { Respiratory } \\
\text { department }\end{array}$ & 145 & 14 & 9.66 & 40 & 12 & 30 & 52 & 12 & 23.08 & 116 & 22 & 18.9 \\
\hline $\begin{array}{l}\text { Other non-ICU } \\
\text { departments }\end{array}$ & 2707 & 207 & 7.65 & 589 & 228 & 38.71 & 384 & 94 & 24.48 & 484 & 100 & $20.6 \epsilon$ \\
\hline$x^{2}$ & 190.766 & & & 97.642 & & & 75.078 & & & 69.515 & & \\
\hline$P$ & $<0.001$ & & & $<0.001$ & & & $<0.001$ & & & $<0.001$ & & \\
\hline Total & 6584 & 704 & 10.69 & 1264 & 554 & 43.83 & 1115 & 376 & 33.72 & 1752 & 475 & 27.11 \\
\hline $\begin{array}{l}\text { All departments } \\
\text { in the hospital }\end{array}$ & 13704 & 3474 & 25.35 & 2123 & 1093 & 51.48 & 5943 & 4704 & 79.15 & 4365 & 2051 & $46.9 \subseteq$ \\
\hline$x^{2}$ & 584.309 & & & 15.583 & & & 960.632 & & & 203.726 & & \\
\hline$P$ & $<0.001$ & & & $<0.001$ & & & $<0.001$ & & & $<0.001$ & & \\
\hline
\end{tabular}

Table 3 Distribution of MDRO infection sites in non-ICU departments 


\begin{tabular}{|lll|}
\hline Infection site & Strains $(\mathrm{n}=1116)$ & constituent ratio (\%) \\
\hline Lower respiratory tract & 540 & $48.39 \%$ \\
\hline Surgical site & 132 & $11.83 \%$ \\
\hline Urinary tract & 123 & $11.02 \%$ \\
\hline Skin soft-tissue infection & 104 & $9.32 \%$ \\
\hline Bloodstream infection & 104 & $9.32 \%$ \\
\hline Abdominal (pelvic) cavity tissue infection & 45 & $4.03 \%$ \\
\hline Other site & 9 & $0.81 \%$ \\
\hline Nasosinusitis & 8 & $0.72 \%$ \\
\hline Oral cavity (mouth, tongue, gums) & 8 & $0.72 \%$ \\
\hline Ascites & 7 & $0.63 \%$ \\
\hline Omphalitis & 7 & $0.63 \%$ \\
\hline Meningitis, ventriculitis & 6 & $0.54 \%$ \\
\hline Upper respiratory tract & 5 & $0.45 \%$ \\
\hline Burn infection & 3 & $0.27 \%$ \\
\hline Otitis externa, otitis media & 3 & $0.27 \%$ \\
\hline Soft tissue infection & 2 & $0.18 \%$ \\
\hline Other & 10 & $0.90 \%$ \\
\hline Total & 1116 & $100 \%$ \\
\hline
\end{tabular}

\section{Figures}




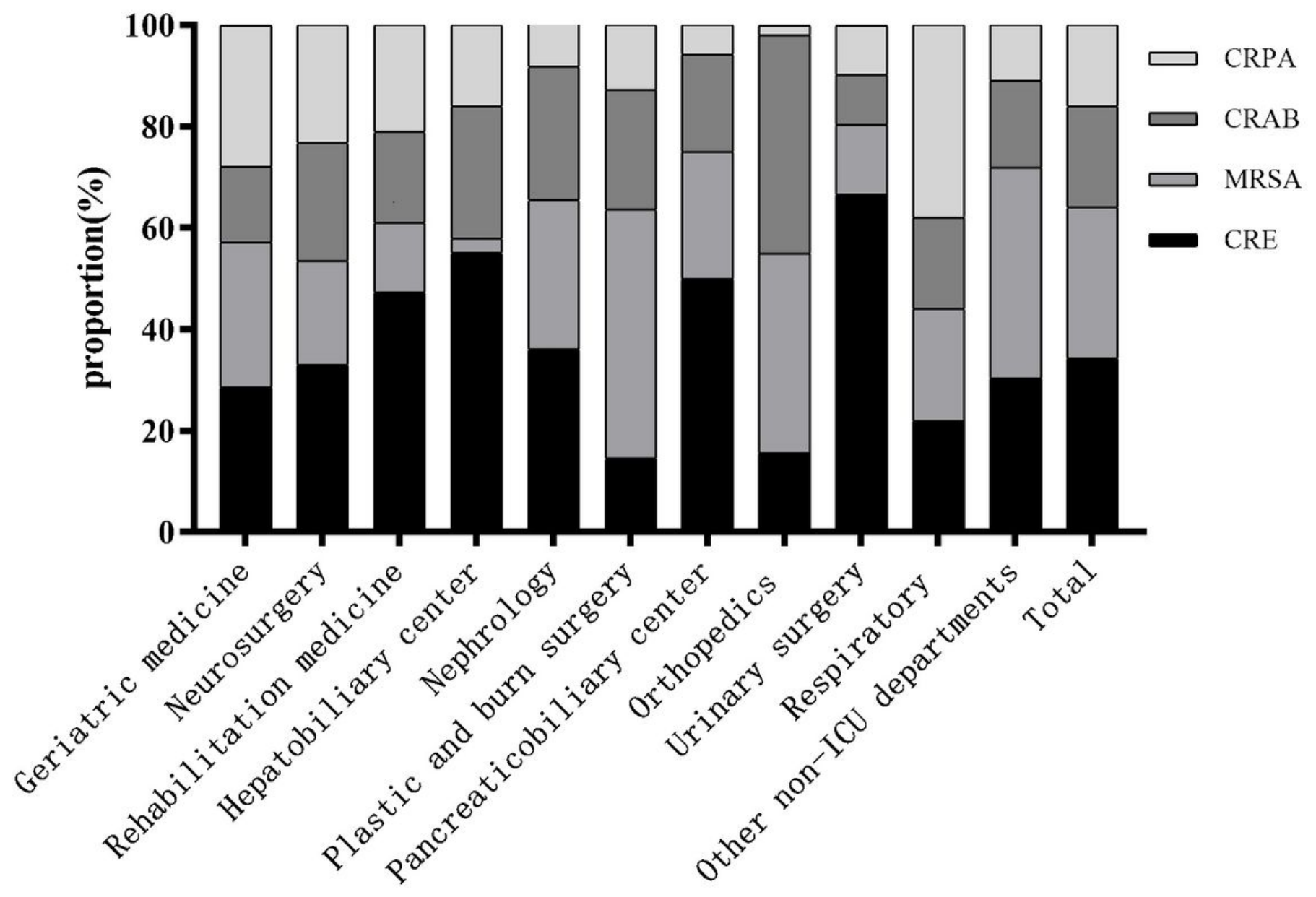

Figure 1

the proportion of CRPA infection was high in respiratory department (38.00\%). The details were shown in Figure 1. 

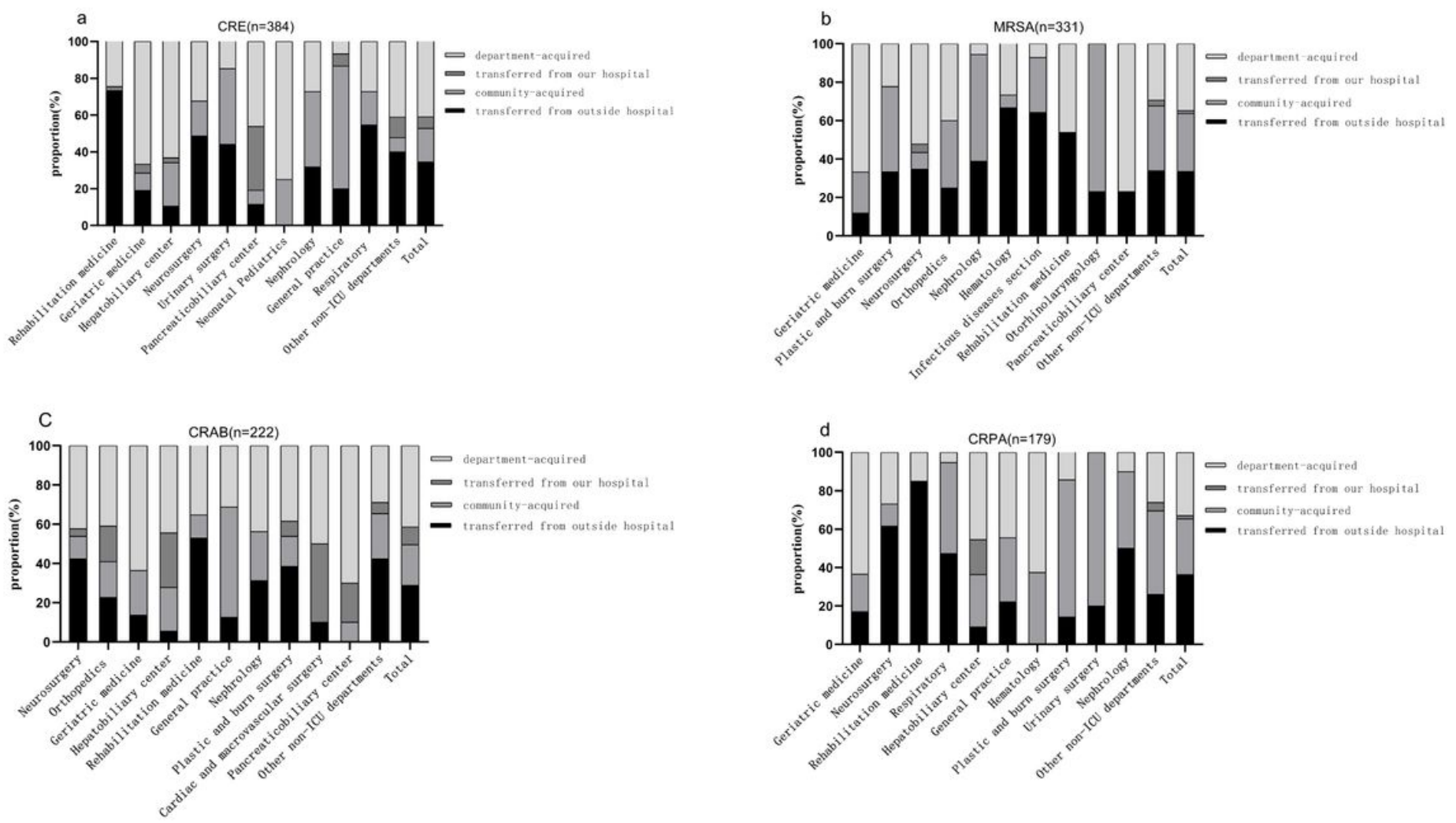

Figure 2

The proportions of out-of-hospital infection of CRE (Fig. 2a), MRSA (Fig. 2b), CRAB (Fig. 2c) and CRPA (Fig. 2d) 